\title{
The therapeutic effects of music in children following cardiac surgery
}

\author{
Thamine P. Hatem, ${ }^{1}$ Pedro I. C. Lira, ${ }^{2}$ Sandra S. Mattos ${ }^{3}$
}

\begin{abstract}
Objective: To investigate, both objectively and subjectively, the effect of music on children in a pediatric cardiac intensive care unit following heart surgery, in conjunction with standard care.

Methods: Randomized clinical trial with placebo, assessing 84 children, aged 1 day to 16 years, during the first 24 hours of the postoperative period, given a 30 minute music therapy session with classical music and observed at the start and end of the session, recording heart rate, blood pressure, mean blood pressure, respiratory rate, temperature and oxygen saturation, plus a facial pain score. Statistical significance was set at $5 \%$.

Results: Five of the initial 84 patients (5.9\%) refused to participate. The most common type of heart disease was acyanotic congenital with left-right shunt ( $41 \%$ of cases: $44.4 \%$ of controls). Statistically significant differences were observed between the two groups after the intervention in the subjective facial pain scale and the objective parameters heart rate and respiratory rate $(p<0.001, p=0.04$ and $p=0.02$, respectively).

Conclusions: A beneficial effect from music was observed with children during the postoperative period of heart surgery, by means of certain vital signs (heart rate and respiratory rate) and in reduced pain (facial pain scale). Nevertheless, there are gaps to be filled in this area, and studies in greater depth are needed.
\end{abstract}

J Pediatr (Rio J). 2006;82(3):186-92: Music therapy, postoperative period, heart surgery, children, intensive care.

\section{Introduction}

Music is a combination of rhythmical, harmonic and melodic sounds, and many peoples, throughout history, have believed in its medicinal effects. ${ }^{1}$ Music therapy is a systematic intervention process in which a therapist helps patients to improve health, utilizing musical experiences and the relationships that develop through them, such as dynamic forces of change. It is a multidisciplinary process in which one uses, basically, music as the primary element of work. ${ }^{1}$ The idea of music with therapeutic effects, affecting human health and behavior, is as ancient as the writings of Aristotle and Plato. Music has been used

1. Mestre, Universidade Federal de Pernambuco (UFPE), Recife, PE, Brasil. Unidade de Cardiologia e Medicina Fetal (UCMF), Recife, PE, Brasil.

2. Doutor, UFPE, Recife, PE, Brasil.

3. Especialista em Cardiologia Pediátrica, UCMF, Recife, PE, Brasil.

Manuscript received Dec 152005, accepted for publication Feb 082006.

Suggested citation: Hatem TP, Lira PI, Mattos SS. The therapeutic effects of music in children following cardiac surgery. J Pediatr (Rio J). 2006;82:186-92. therapeutically for centuries, and there are numerous examples of music's curative and preventative powers, in many historical documents from different cultures. ${ }^{2}$

At the turn of the century, there was renewed interest in the effects of music on health, to a great extent due to the emphasis given the search for pain control. ${ }^{3-5}$ Pain is an experience that many patients have in common, irrespective of age, type of disease or event. Some authors have studied the effects of music for pain control and demonstrated a reduction in the perception of pain after the institution of music therapy. ${ }^{3,6}$ Its mechanism of action is still very controversial and several theories have been offered to explain it, such as that music acts on autonomic function, stimulating the pituitary, resulting in the liberation of endorphin (natural opioid), reducing pain and leading patients who receive music therapy to potentially reduce analgesic requirements. There also appears to be a reduction in the liberation of catecholamines, which could explain the reductions in heart rate (HR) and blood pressure (BP). 1,6 
Another phenomenon that has been of importance to the resurgence of musical interventions in health is anxiety. Around 70 to $87 \%$ of patients in intensive care units (ICU) suffer from anxiety, ${ }^{1}$ which is commonly associated with agents of stress, such as disease and hospitalization, in addition to being significantly increased if related to the individual themselves or related to heart problems. ${ }^{7,8}$

Traditionally, treatment of heart conditions is focused on the pathophysiologic needs of the patient - control of pain and discomfort, good tissue perfusion and monitoring of cardiac disturbances plus rest. ${ }^{9}$ It is in these situations that the presence of rhythmical and harmonious sounds can relieve pain of both physical and emotional origins ${ }^{10}$ and act on hemodynamic parameters, such as HR, BP and temperature, ${ }^{9,11}$ relaxing patients by regulating breathing, relaxing muscles and improving sleep. $4,12,13$

Congenital heart disease is a common malformation with an with incidence of 8-10 for each 1,000 live births. According to the Brazilian Institute of National Statistics and Geography (IBGE - Instituto Brasileiro de Geografia e Estatística), there are approximately 350,000 births per year in the state of Pernambuco and more than 3,000 of these children are born with heart disease. A large proportion of these children (50-60\%) will need surgery during childhood.

Observing the good results of the introduction of musical techniques to pediatric, neonatal, and adult ICUs and coronary units, $8,12,14,15$ and considering the high incidence and prevalence of childhood congenital heart disease that requires surgery, we decided to objectively verify the effect of music on HR, BP, temperature $(T)$, mean blood pressure (MBP), respiratory rate (RR) and oxygen saturation $\left(\mathrm{SatO}_{2}\right)$, during the immediate postoperative period of pediatric heart surgery patients and to subjectively assess music for pain control, used in conjunction with therapeutic measures already used in conventional medical practice.

\section{Methods}

This was a randomized clinical trial, controlled by a placebo, which, in this case, was the absence of music. Eighty-four consecutive children, aged 1 day to 16 years (the specific age range of the ICU), were studied during the immediate postoperative period of heart surgery, defined as the first 24 hours after surgery. This is an ICU exclusively dedicated to pediatric cardiac patients, part of the Hospital do Coração, Real Hospital Português de Pernambuco, and the study took place from January to June 2004.

Information from a pilot study was used to perform a sample size calculation, adopting an alpha error of $5 \%$ and power of $80 \%$. An intervention-control ratio of three to one was defined and it was estimated that the percentage of patients in the control group scoring two or more on the facial pain scale at the end of the study would be $65 \%$, and that for the exposure group (intervention), this percentage would be $25 \%$, which resulted in a sample estimate of 18 controls and 54 exposed subjects.

After surgery, and with the prior consent of parents by means of a free and informed consent form, children were randomized systematically (three consecutive interventions, then one control) and given either 30 minute music therapy session with the classical music that had been chosen in advance (Spring, from Vivaldi's Four Seasons). The music was chosen based on previous studies that have demonstrated that relaxing music (smooth classical music) is composed of low amplitudes, simple and direct rhythms and a frequency (tempo) of approximately 60 to 70 beats per minute. ${ }^{8,16}$ Music was reproduced through Philips SBC HL120 headphones, coupled to a Philips Power Saving 12 ESP 3 CD player. It is particularly important to use headphones in intensive care because they allow the patient to focus on the music rather than the noises emitted by the units' equipment and without disturbing other patients and or health professionals.

The variables $\mathrm{HR}, \mathrm{BP}, \mathrm{MBP}, \mathrm{RR}, \mathrm{T}$ and $\mathrm{SatO}_{2}$, were assessed using a Siemens SC 6002 XL cardiopulmonary monitor, before playing the music and again 30 minutes after (the optimum duration is not known, but studies suggest a range of 25 to 90 minutes). ${ }^{8,16}$ Other variables were also observed, including age, sex, type of heart disease and severity of heart surgery (Jenkins et al. criteria), 17 and correlations with other variables investigated. Patients were classed by type of heart disease as follows: acyanotic congenital heart disease (ACHD) with left-right shunt; obstructive ACHD; cyanotic congenital heart disease (CCHD) with pulmonary hypoflow; CCHD with pulmonary hyperflow; complex congenital heart disease (CHD)' and acquired heart diseases.

The controls were observed under the same conditions

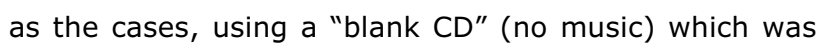
"played" for the same 30 minutes, with data collected before and afterwards.

Certain precautions were taken to improve the accuracy of measurements. The observer was required to follow a data collection manual containing useful information to ensure correct execution of the activities and had previously attended a trained lecture on the evaluation method (standardization). Data collection was performed using a standardized form, which was completed by the nursing auxiliary responsible for the child, and who also performed a subjective facial pain scale assessment ${ }^{18}$ during the first and last minutes of the music therapy session. Photographs were taken of the patients (with their guardians' prior consent) at the point of intervention to enable comparative observation of what was recorded by the observer and 
what had been learnt at the lecture. Drugs being given at the time of the experiment were noted for later assessment of possible exclusions due to interference. The volume (in decibels) was calculated for each child in advance using a decibel meter This ruled out the possibility of auditory damage, particularly of newborn infants. A pilot study was run to improve systematization and instrument checks in the current experiment.

Data were double input using Epi-Info version 6.04d and SPSS version 8.0 was used for statistical analysis. Statistical analyses were performed of measures of central tendency and chi-square and Fisher tests were used to compare frequencies and the Wilcoxon test was used to tests differences in medians. The level of significance adopted was $5 \%$ for all tests.

In accordance with resolution 196 by the Research Ethics Committee, the study was performed after a favorable hearing by the committee at the Hospital das Clínicas, Universidade Federal de Pernambuco (process no. 031/2004 - CEP/CCS).

\section{Results}

Eighty-four patients were studied during the 6-month period, being 63 cases (with music therapy) and 21 controls (without music therapy). Five (5.9\%) children were excluded: three from the control group and two from the intervention group, leaving 79 children, giving a intervention-control ratio of $3.4: 1$.

Groups were analyzed for age and sex distribution, and uniformity was observed. There was a mild predominance of females in the no music therapy group (control) and of males in the group with music therapy (intervention), but without statistically significant differences between the groups ( $p=0.45)$. Groups were analyzed in three age groups, with the highest numbers being in the 1 to 6 years range. The differences between groups were not significant ( $p=0.33$ ).

The form of heart disease that predominated was ACHD with left-right shunt, accounting for approximately $40 \%$ in both groups. (Table 1 ).

Patients were further classified by type of surgery, for which the Jenkins et al. ${ }^{17}$ severity-based classification was used. This distribution can be observed in Table 2 .

Table 3 lists the scores for the two groups on the Bieri et al. ${ }^{18}$ facial pain scale, before and after the music therapy intervention. A statistically significant difference is observed between the two groups at the end of the intervention $(p<0.001)$. The scale originally contains seven faces depicting expressions in ascending order of pain.

Table 4 lists the characteristics of the two groups in terms of $\mathrm{HR}, \mathrm{MBP}, \mathrm{SBP}, \mathrm{DBP}, \mathrm{RR}$, SatO $\mathrm{O}_{2}$ and $\mathrm{T}$, before and after intervention. Before the intervention, there were no statistically significant differences in any of the variables mentioned. After the intervention, there were significant difference between the groups, with lower HR and RR among children given music therapy when compared with those without music therapy $(p=0.04$ and $p=0.02$, respectively). The remaining variables did not exhibit any statistically significant differences.

\section{Discussion}

Just five from the total of 84 children were excluded: three controls, because of refusal due to there being no sound on the $C D$, which is understandable since these were older children who thought the $C D$ player was

Table 1 - Distribution of heart diseases across music therapy and control groups during the postoperative period of heart surgery

\begin{tabular}{|c|c|c|c|c|c|c|}
\hline \multirow{3}{*}{ Type } & \multicolumn{4}{|c|}{ Group } & & \\
\hline & \multicolumn{2}{|c|}{ No music therapy } & \multicolumn{2}{|c|}{ Music therapy } & \multicolumn{2}{|c|}{ Total } \\
\hline & $\mathbf{n}$ & $\%$ & $\mathbf{n}$ & $\%$ & $\mathbf{n}$ & $\%$ \\
\hline Left-right shunt ACHD & 8 & 44.4 & 25 & 41.0 & 33 & 41.8 \\
\hline Obstructive ACHD & - & 0 & 5 & 8.2 & 5 & 6.3 \\
\hline CCHD with hypoflow & 5 & 27.8 & 11 & 18.0 & 16 & 20.3 \\
\hline CCHD with hyperflow & - & 0 & 2 & 3.3 & 2 & 2.5 \\
\hline Complex CHD & 4 & 22.2 & 14 & 23.0 & 18 & 22.8 \\
\hline Acquired HD & 1 & 5.6 & 4 & 6.6 & 5 & 6.3 \\
\hline Total & 18 & 22.8 & 61 & 77.2 & 79 & 100.0 \\
\hline
\end{tabular}

$\mathrm{ACHD}=$ acyanotic congenital heart disease $\mathrm{CCHD}=$ cyanotic congenital heart disease $\mathrm{CHD}=$ congenital heart disease; $\mathrm{HD}=$ heart disease. 
Table 2 - Type of surgery (Jenkins ascending severity classification) by intervention or not with music therapy in children in the postoperative period of heart surgery

\begin{tabular}{|c|c|c|c|c|c|c|}
\hline \multirow{3}{*}{ Jenkins } & \multicolumn{4}{|c|}{ Group } & & \\
\hline & \multicolumn{2}{|c|}{ No music therapy } & \multicolumn{2}{|c|}{ Music therapy } & \multicolumn{2}{|c|}{ Total } \\
\hline & $\mathbf{n}$ & $\%$ & $\mathbf{n}$ & $\%$ & $\mathbf{n}$ & $\%$ \\
\hline 1 & 7 & 38.9 & 23 & 37.7 & 30 & 38.0 \\
\hline 2 & - & 0.0 & 6 & 9.8 & 6 & 7.6 \\
\hline 3 & 8 & 44.4 & 27 & 44.3 & 35 & 44.3 \\
\hline 4 & 3 & 16.7 & 5 & 8.2 & 8 & 10.1 \\
\hline Total & 18 & 22.8 & 61 & 77.2 & 79 & 100.0 \\
\hline
\end{tabular}

Table 3 - Comparison between number of faces before and after intervention with music therapy or placebo in children in the postoperative period of heart surgery

\begin{tabular}{|c|c|c|c|c|c|c|c|c|c|c|}
\hline \multirow{3}{*}{ Faces } & \multicolumn{4}{|c|}{ Before } & \multicolumn{6}{|c|}{ After } \\
\hline & \multicolumn{2}{|c|}{ No music therapy } & \multicolumn{2}{|c|}{ Music therapy } & \multirow[b]{2}{*}{$\mathbf{p}$} & \multicolumn{2}{|c|}{ No music therapy } & \multicolumn{2}{|c|}{ Music therapy } & \multirow[b]{2}{*}{$\mathbf{p}$} \\
\hline & $\mathbf{n}$ & $\%$ & $\mathbf{n}$ & $\%$ & & $\mathbf{n}$ & $\%$ & $\mathbf{n}$ & $\%$ & \\
\hline 1 & 6 & 33.3 & 10 & 16.4 & & 3 & 16.7 & 47 & 77.0 & \\
\hline 2 & 8 & 44.5 & 25 & 41.0 & & 8 & 44.4 & 14 & 23.0 & \\
\hline 3 & 2 & 11.1 & 13 & 21.3 & & 7 & 38.9 & 0 & 0.0 & \\
\hline$\geq 4$ & 2 & 11.1 & 13 & 21.3 & & - & - & - & - & \\
\hline Total & 18 & 100.0 & 61 & 100.0 & 0.32 & 18 & 100.0 & 61 & 100.0 & $<0.001$ \\
\hline
\end{tabular}

broken, and two cases, who refused due to the style of the music (classical). These older children already had welldefined personal taste in music, which impeded the use of classical music.

With respect of the use of sedative or positive inotropic drugs and respirator settings, there were no reports of these needing modification during the experiment in either group, which leads us to believe that these variables did not exert an influence on the study parameters.

There was no difference in the distribution of the sexes in the two groups, which is indicative of correct random group allocation. This is in common with another study with the same characteristics, 19 where no significant difference were observed between the two groups assessed, and also with other work in the area in terms of the age groups most affected.19,20
The distribution of type of heart disease in the current study was also similar to what is found in the literature, where left-right shunt heart diseases are the most common $(41.8 \%)$, with intraventricular communication (IVC) being the most common of these. Group 3, CHD with pulmonary hypoflow $(20.3 \%)$, is of note, with emphasis on the inclusion of tetralogy of Fallot, considered by many authors as the most common CHD. ${ }^{19,20}$ Also of note is group 5 $(22.8 \%)$, complex heart disease, a pattern that is common at referral centers for pediatric heart surgery, as is the case at the study center. ${ }^{19-21}$

The Jenkins et al. classification has shown itself highly suitable for classifying surgery in a scale of ascending severity. It was introduced in $1995 .{ }^{17}$ The results show a predominance of Jenkins classes $1(38.0 \%)$ and $3(44.3 \%)$, which is in common with findings at other centers with 
Table 4 - Comparison of the variables HR, MBP, BP, RR, SATO and $T$ before and after intervention or not with music therapy in children in the postoperative period of heart surgery

\begin{tabular}{|c|c|c|c|c|c|}
\hline \multirow[t]{2}{*}{ Variables } & \multicolumn{2}{|c|}{ No music therapy $(n=18)$} & \multicolumn{2}{|c|}{ Music therapy $(n=61)$} & \multirow[t]{2}{*}{ p* } \\
\hline & Median & $\left(I Q_{25-75 \%}\right)$ & Median & $\left(I Q_{25-75 \%}\right)$ & \\
\hline \multicolumn{6}{|l|}{$\mathrm{HR}$} \\
\hline Before & 122.5 & $(115.0-144.0)$ & 126.0 & $(106.0-138.0)$ & 0.54 \\
\hline After & 131.5 & $(121.0-149.0)$ & 121.0 & $(104.0-137.0)$ & 0.04 \\
\hline \multicolumn{6}{|l|}{ MBP } \\
\hline Before & 80.5 & $(73.0-91.0)$ & 87.0 & $(71.0-97.0)$ & 0.38 \\
\hline After & 83.0 & $(73.0-91.0)$ & 80.0 & $(72.0-93.0)$ & 0.88 \\
\hline \multicolumn{6}{|l|}{ SBP } \\
\hline Before & 101.5 & $(90.0-112.0)$ & 110.0 & $(93.0-121.0)$ & 0.33 \\
\hline After & 106.2 & $(94.0-121.0)$ & 104.0 & $(84.5-111.0)$ & 0.22 \\
\hline \multicolumn{6}{|l|}{ DBP } \\
\hline Before & 62.0 & $(53.0-75.0)$ & 69.0 & $(56.0-80.0)$ & 0.28 \\
\hline After & 62.0 & $(58.0-68.0)$ & 66.0 & $(57.0-74.5)$ & 0.52 \\
\hline \multicolumn{6}{|l|}{$\mathrm{RR}$} \\
\hline Before & 32.0 & $(26.0-42.0)$ & 30.0 & $(24.0-38.0)$ & 0.42 \\
\hline After & 34.0 & $(28.0-40.0)$ & 28.0 & $(23.0-36.0)$ & 0.02 \\
\hline \multicolumn{6}{|l|}{$\mathrm{SatO}_{2}$} \\
\hline Before & 98.0 & $(97.0-100.0)$ & 100.0 & $(98.0-100.0)$ & 0.44 \\
\hline After & 98.0 & $(94.0-100.0)$ & 100.0 & $(97.0-100.0)$ & 0.13 \\
\hline \multicolumn{6}{|l|}{$\mathrm{T}$} \\
\hline Before & 36.5 & $(36.0-37.0)$ & 36.3 & $(36.0-36.8)$ & 0.29 \\
\hline After & 36.5 & $(36.2-37.0)$ & 36.1 & $(36.0-36.6)$ & 0.07 \\
\hline
\end{tabular}

* Wilcoxon test.

$\mathrm{BP}=$ blood pressure; $\mathrm{DBP}=$ diastolic blood pressure; $\mathrm{HR}=$ heart rate; $\mathrm{IQ}=$ interquartile; $\mathrm{MBP}=$ mean blood pressure; $\mathrm{RR}=$ respiratory rate $; \mathrm{SBP}=$ systolic blood pressure $; \mathrm{SatO}_{2}=$ oxygen saturation; $\mathrm{T}=$ temperature.

similar profiles in terms of coverage and complexity. Attention is drawn to a mild predominance of class 3, which is possible due to the fact that the class includes surgery to correct the tetralogy of Fallot, the principal cyanotic heart disease in our area, and Blalock-Taussing palliative surgery and other shunts that are often used at our service as palliative treatment for complex heart disease. ${ }^{19,21}$

As has been observed, the groups (with and without music therapy) were homogenous at the start of the procedure in terms of the variables studied, confirming the random nature of the groups.

The two groups were subjectively assessed with a facial pain scale, ${ }^{18}$ before and after the music therapy session. This scale is most suitable to assessing a highly heterogenous group, such as newborn infants, who are unable to give their own opinions. This need for heterogeneity makes comparison between this study and others problematic, since they refer to pre-established scores or scales based on the patient's own opinion and not via third parties. Nevertheless, once possible bias had been ruled out, we observed a statistically significant difference between the two groups, which appears to demonstrate that the music acted to reduce the pain and anxiety of patients during the immediate postoperative period. Similar findings were observed through different means of assessment (scores and questionnaires) in published literature. 22,23

This study's findings with respect to the physiological aspect of HR are in agreement with other investigations that have also registered reduction, $8,22,24$ which shows that music is contributing to reduce the anxiety of small patients in ICU during the postoperative period for CHD, which situation itself increases stress. A study in China failed to observe any effect by music on $H R$, but this may be the result of a small sample size and sociocultural 
features of the Chinese population, as the author suggests. 25

Some studies have demonstrated reduced MBP, which is in contrast with the results of this study. Nevertheless, inconsistencies in the results of clinical trials with respect of physiological effects have been observed before, which puts emphasis on the need for studies into the influence of musical elements, such as rhythm, tempo, harmony and timbre have on these parameters. 26 Published literature already contains reports of reductions in systolic pressure, ${ }^{12}$ which we also failed to observe in this study, perhaps because of the small sample size or perhaps because of the type of postoperative period (heart surgery), where chronotropic drugs are often used continuously, explaining the fact that pressure remained similar before and after

Some studies 22,24 agree with our finding that RR reduced. However, a study in China ${ }^{25}$ did not observe any effect on RR from music with patients post catheterization, which could have been the result of the small sample size in the pilot study.

Previous studies have demonstrated alterations in $\mathrm{SatO}_{2}$ and $\mathrm{T}$ (up and down, respectively), as a result of music. $8,27-29$ This was not confirmed in the current study, nor by other experiments in the area, 24,30 which indicates there is a need to carry out further studies to better observe the role that music therapy has in these physiological effects.

Extrapolation of this study is prejudiced by the sample size, by there only existing one specialist hospital in this area at the time of data collection. Another methodological feature is that the study subjects were not randomized by age group, due to the heterogeneous nature of the patients at the center. Another limitation is related to the subjective method of analysis of the pain scale. Attempts were made to limit any bias using an explanatory lecture with similar faces to unify the experiment, which does not guarantee its homogeneity. Perhaps more observers for each face would be better. Another possible limitation is the use of cardiovascular and sedative drugs, which, even though they were not modified during the experiment (30 minutes), could in some way impede more faithful assessment of each patient. Another limitation of great importance in the musical taste of each patient, which, in this study, was not taken into account and which is of great importance for patient acceptance. This choice made in advance by the researcher was made in an attempt to homogenize as much as possible the patients studied. The use of a blank CD with the controls made acceptance difficult with older children, since they refused to listen to a CD with no music whatsoever. Overall, these limitations do not invalidate the study and are points to think about in any new assessment.

\section{Final considerations}

This research has demonstrated the beneficial action of music on children in the postoperative period for heart surgery, through certain vital signs (HR and RR), and, subjectively, in reductions in pain (facial pain scale). Notwithstanding, there are a number of gaps to be filled in this area, and we believe there is a need for a more specific study, in greater depth, into the elements of music (rhythm, tempo, harmony and timbre) and into making it more suitable for each individual, their needs and tastes.

\section{Acknowledgements}

Thanks to the children and their families and the fieldwork team for their participation. To the Fetal Cardiology and Medicine Unit and Hospital do Coração, Real Hospital Português de Pernambuco for their support. Also to the CNPq for their financial support to Prof. Pedro Lira.

\section{References}

1. Henry LL. Music therapy: a nursing intervention for the control of pain and anxiety in the ICU. A review of the research literature. Dimens Crit Care Nurs. 1995;14:295-304.

2. Grant R. Music therapy assessment for developmentally disabled clients. In: Wigram T, Saperston B, West R, editors. The art and science of music therapy: a handbook. London: Harwood Academic; 1995. p. 273-87.

3. Heitz L, Symreng T, Scamman FL. Effect of music therapy in the postanesthesia care unit: a nursing intervention. J Post Anesth Nurs. $1992 ; 7: 22-31$.

4. Good M, Stanton-Hicks M, Grass JA, Anderson GC, Lai H-L, Roykulcharoen $\mathrm{V}$, et al. Relaxation and music to reduce postsurgical pain. J Adv Nurs. 2001;33:208-15.

5. Standley JM. A meta-analysis of the efficacy of music therapy for premature infants. J Pediatr Nurs. 2002;17:107-13.

6. Whipple B, Glynn NJ. Quantification of the effects of listening to music as a noninvasive method of pain control. Sch Inq Nurs Pract. 1992;6:43-58.

7. Mok $\mathrm{E}$, Wong $\mathrm{K}-\mathrm{Y}$. Effects of music on patient anxiety. AORN J. 2003;77:396-7, 401-6, 409-10.

8. Guzzeta C. Effects of relaxation and music therapy on patients in a coronary care unit with presumptive acute myocardial infarction. Heart Lung. 1989;18:609-16.

9. Thelan LA, Davie JK, Urden LD. Text book of critical care nursing: diagnosis and management. St. Louis: CV Mosby; 1990.

10. Cassem NH, Hackett TP. Psychiatric consultation in a coronary care unit. Ann Intern Med. 1971;75:9-14.

11. Jillings $C R$. The experience of acute illness: impact on psychosocial needs. Crit Care Nurse. 1985;5:94.

12. Updike P. Music therapy results for ICU patients. Dimens Crit Care Nurs. 1990;9:39-45.

13. Kain ZN, Wang SM, Mayes LC, Krivutza DM, Teague BA. Sensory stimuli and anxiety in children undergoing surgery: A randomized, controlled trial. Anesth Analg. 2001;92:897-903.

14. Heitz L, Symreng T, Scamman FL. Effect of music therapy in the postanesthesia care unit: a nursing intervention. J Post Anesth Nurs. 1992; 7:22-31.

15. Marconato C, Munhoz EC, Menim MM, Albach MT. Application of receptive music therapy in internal medicine and cardiology. Arq Bras Cardiol. 2001;77:138-41.

16. White JM. Music therapy helps reduce anxiety in the myocardial infarction patients. Clin Nurse Spec. 1992;6:58-63. 
17. Jenkins $K L$, Newburger JW, Lock JE, Davis RB, Coffman GA, Iezzoni LI. In-hospital mortality for surgical repair of congenital heart defect: preliminary observation of variation by hospital caseload. Pediatrics. 1995;95:323-30.

18. Bieri D, Reeve RA, Champion GD, Addicoat L, Ziegler JB. The faces pain scale for the self-assessment of the severity of pain experienced by children: development, initial validation, and preliminary investigation for ratio scale properties. Pain. 1990;41:139-50

19. Hannan EL, Racz M, Kavey RE, Quaegebeur JM, Williams R. Pediatric cardiac surgery: the effect of hospital and surgeon volume on in-hospital mortality. Pediatrics. 1998;101:963-9.

20. Miyague NI, Cardoso SM, Meyer F, Utramari FT, Araujo FH, Rozkowisk I, et al. Estudo epidemiológico de cardiopatias congênitas na infância e adolescência. Análise em 4538 Casos. Arq Bras Cardiol. 2003;80:269-73.

21. Stark J, Gallivan S, Lovegrove J, Hamilton JR, Monro JL, Pollock JC, et al. Mortality rates after surgery for congenital heart defects in children and surgeons' performance. Lancet. 2000;355:1004-7.

22. White JM. Effects of relaxing music on cardiac autonomic autonomic balance and anxiety after acute myocardial infarction. Am J Crit Care. 1999;8:220-30.

23. Wong HL, Lopez-Nahas V, Molassiotis A. Effects of music therapy on anxiety in ventilator-dependent patients. Heart Lung. 2001;30:376-87.

24. Chlan LL. Psychophysiologic responses of mechanically ventilated patients to music: a pilot study. Am J Crit Care. 1995;4:233-8.
25. Taylor-Piliae RE, Chair SY. The effect of nursing interventions utilizing music therapy or sensory information on Chinese patients anxiety prior to cardiac catheterization: a pilot study. Eur J Cardiovasc Nurs. 2002;1:203-11.

26. Wigran AL. The effects of vibroacoustic therapy on clinical and non-clinical populations [dissertation]. London: London University; 1996.

27. Evans D. The effectiveness of music as an intervention for hospital patients: a systematic review. J Adv Nurs. 2002;37:8-18.

28. Kibler VE, Rider MS. Effects of progressive muscle relaxation and music on stress as measured by finger temperature response. J Clin Psychol. 1983;39:213-5.

29. Chou LL, Wang RH, Chen SJ, Pai L. Effects of music therapy on oxygen saturation in premature infants receiving endotracheal suctioning. J Nurs Res. 2003;11:209-16.

30. Cadigan ME, Caruso NA, Haldeman SM, McNamara ME, Noyes DA, Spadafora MA, et al. The effects of music on cardiac patients on bed rest. Prog Cardiovasc Nurs. 2001;16:5-13.

\section{Correspondence:}

Thamine de Paula Hatem

Rua Padre Roma, 688/202, Parnamirim

CEP 52060-060 - Recife, PE - Brazil

Tel.: +55 (81) 3268.6025

Fax: + 55 (81) 3231.0490

E-mail: thamine@gmail.com 